\title{
HOW COMMON IN-CAR DISTRACTIONS AFFECT DRIVING PERFORMANCE IN SIMPLE AND COMPLEX ROAD ENVIRONMENTS
}

\author{
Robert J. Nowosielski \& Lana M. Trick \\ University of Guelph, Guelph, Ontario, Canada \\ Email: rnowosie@uoguelph.ca, ltrick@uoguelph.ca
}

\begin{abstract}
Summary: Distracted driving (driving while performing a secondary task) is the cause of many collisions. Although the research has stressed the deleterious effects of distraction, there may be situations where distraction improves driving performance. Boredom is associated with collision risk, and it is possible that some types of secondary task may combat boredom on simple monotonous drives. In this study, licensed drivers were tested in a driving simulator (a car body surrounded by screens) that simulated simple or complex roads. Road complexity was manipulated by increasing traffic, scenery, and the number of curves in the drive. Participants either drove (single task), or they drove while listening to an audiobook or having a hands-free cellular phone conversation. Driving performance was measured in terms of speed, standard deviation of speed, standard deviation of lateral position (SDLP), and hazard response times. Task condition and road complexity had no significant effect on driving speed or standard deviation of speed. There was a trend to greater SDLP on the simple drives, where there was little oncoming traffic, though this was only statistically significant in the Audiobook condition. However, there was also evidence that audiobooks could be beneficial. On simple roads, drivers listening to audiobooks had significantly faster hazard response times that those that were driving (single task) or driving while having a hands-free conversation, though this pattern of response was not evident on complex drives. These results suggest that audiobooks could play a role in helping drivers stay focused on monotonous drives.
\end{abstract}

\section{OBJECTIVES}

Secondary tasks (distractions) are a factor in many collisions (Lee, Champagne, \& Francescutti, 2013). Although, many studies focus on the deleterious effects of cell phones, there are many other activities that might divert a drivers' attention. This includes talking to a passenger, texting, using GPS, and adjusting a media console (Horberry, Anderson, Regan, Triggs, \& Brown, 2006). These various distractions may vary in terms of their impact on driving performance. Some incar distractions like texting or talking on the phone, have been found to significantly increase the risk of collision, although, some of the cell phone studies are now showing that hands-free cell phones are less disruptive. Others distractions, such as listening to the radio, have shown to have no impact on driving performance (Bergmark et al., 2016). The extent to which a distraction interferes with driving performance depends on how much the task contributes to the driver's cognitive load, which refers to the amount of mental resources (attention, working memory) that an individual has for a task (Palinko \& Kun, 2012). The more a secondary task contributes to a driver's cognitive load, the more driving performance tends to suffer (Lee, Lee, \& Boyle, 2007). 
The complexity of the driving environment is another factor contributes significantly to a driver's cognitive load. An urban environment with heavy traffic, dense roadside scenery and many potential turns will contribute to a driver's cognitive load much more than a rural environment light traffic, sparse roadside scenery and few turns (Paxion, Galy, \& Berthelon, 2015). Therefore, engaging in secondary tasks while driving in a complex road environment carries a higher risk of collision than doing so in a simple road environment. However, on simple monotonous roads, there is also a risk that drivers may become bored because they are understimulated. When the drive is not sufficiently stimulating, it contributes little to the driver's cognitive load and there is a tendency for the driver's attention to be progressively withdrawn from the driving task (Matthews \& Desmond, 2002). This is especially common on long and repetitive drives such as daily commutes where the familiarity of the environment leads to low arousal and dissatisfaction (Dahlen, Martin, Ragan, \& Kuhlman, 2005). Driving while bored is not as dangerous as distracted driving, but, it may lead the driver to seek stimulation by speeding, engaging in risky maneuvers, or using their mobile phone (Schroeter, Oxtoby, \& Johnson, 2014), and drivers that are both fatigued and bored may fall asleep. Countermeasures such as listening to music and opening the window may not be adequate to alleviate this problem (Schwarz et al., 2012). Unfortunately, although cellular phone conversations may combat boredom, they may also increases the risk of collision (Schwarz et al., 2012).

When drivers listen to an audiobook, it is also a form of secondary task, but to this point, audiobooks have rarely been studied. However, there is some evidence that they may be more cognitively stimulating than listening to the radio, though perhaps less stimulating than having a cell phone conversation (Strayer et al., 2015). Thus, the moderately stimulating effects of audiobooks may be effective at alleviating the effects of boredom that occur on monotonous (under-stimulating) drives. To test this idea, we had participants do three drives over the course of an hour, either on simple (monotonous) straight roads with little scenery or traffic, or on more complex roads with curves, scenery, and traffic. Task condition was varied. Drivers were either required to simply drive, drive while listening to an audiobook, or drive while having a handsfree conversation. Our prediction was that listening to audiobooks in the simple road environment would lead to better driving performance compared to simply driving and having a hands-free conversation, though the effects would not be evident on more complex drives.

\section{METHODS}

\section{Participants}

The participants were 39 licensed drivers recruited from the University of Guelph participant pool ( 22 female; $\mathrm{M}$ age $=18.9$ years, $\mathrm{SD}=1.4)$. All had at least a $\mathrm{G} 2$ license (in Ontario's graduated licensing this permits unsupervised driving on all types of roads) and 6 had a full $\mathrm{G}$ license. Participants were screened to ensure that all were at low risk of Simulator Adaptation Syndrome (dizziness, nausea that some people experience in driving simulators). Of the 39, 19 were randomly assigned to the simple drive and 20 were assigned to the complex drive. 


\section{Design}

This study employed a mixed factorial design with drive type (simple, complex) as the betweensubject variable and task condition (driving with no secondary task, driving during a hands-free cell conversation, driving while listening to an audiobook) as within subject conditions. The dependent variables were hazard response time (HRT), average speed, standard deviation of speed and standard deviation of lane positioning (SDLP).
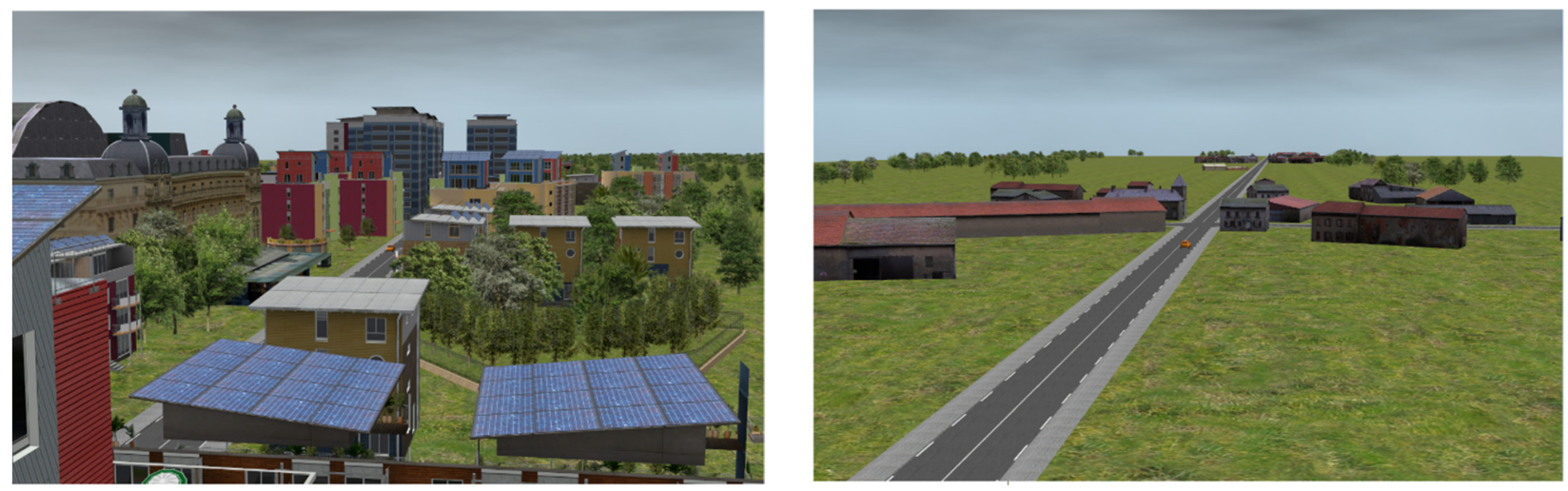

Figure 1. Screenshots of complex road environment (left) and of simple road environment (right)

\section{Apparatus and Stimuli}

Participants were tested in an Oktal driving simulator, which consisted of a fixed base full scale simulator connected to the body of a Pontiac G4 convertible surrounded by 300 degrees of wrap around projector screens. The simulator replicates all the controls that are present in a vehicle including steering, pedals, automatic transmission and a fully interactive media unit that was used to play the audiobooks. The simulator ran on OKTAL's simulator software SCANER Studio 1.5 which allows for full customization of the terrain and events. The simulator was outfitted with a microphone and 4 cameras that were used to monitor the participants for signs of SAS. The data used to calculate hazard reaction was pulled from the simulator, cleaned in Excel, and analyzed in SPSS.

Six 20 -minute drives ( 3 simple, 3 complex) were created, all with posted limits of $90 \mathrm{~km} / \mathrm{h}$. Simple drives involved straight country roads with little traffic and relatively few peripheral visual stimuli (trees and buildings) whereas the complex drives featured moderate oncoming traffic, periodic long gentle curves, and much more peripheral stimulation (10 times more building groups and 200 times more trees). In each drive, there was no other vehicles in the drivers' lane. Hazard response times were based on braking times to 10 peripheral hazards (pedestrians or vehicles that emerged suddenly into the drivers' path from the left or right).

\section{Procedure}

After filling out a demographic questionnaire, participants did a 5-minute practice drive to become accustomed to the driving simulator. Each driver then completed the 3 task conditions. In the driving alone condition, participants simply drove. In the audiobook condition participants listened to an excerpt from the audiobook "Harry Potter and the Deathly Hallows" while driving. 
During the hands-free condition, participants engaged in hands-free conversation with a researcher throughout the drive, on topics such as favorite sports teams, TV shows, and movies. Condition order was counterbalanced. Total testing time was 1.5 hours.

\section{RESULTS}

Mixed factorial analyses of variance were used to analyze the data. The Geisser-Greenhouse correction was applied to the degrees of freedom to ensure against violations of the sphericity assumption. Effect size was assessed using partial eta squared $\left(\eta^{2}\right)$, and Tukey's HSD was used for post-hoc comparisons. Although the roads differed in complexity, comparable straight $1.3 \mathrm{~km}$ sections were used when calculating the average speed, standard deviation in speed, standard deviation of lane position (SDLP), and hazard RT for each task condition.

To begin, we started with analyses of driving speed and standard deviation of speed. As can be seen from Figure 2, drivers adhered fairly closely to the speed limit of $90 \mathrm{~km} / \mathrm{h}$; mixed factorial analyses of variance revealed no main effects $\left(F(1,37)=1.73, n s, \eta^{2}=.09\right)$ or interactions $(F(1$, $\left.37)=2.34, n s, \eta^{2}=.115\right)$. This suggests that there was no sign of compensatory slowing based on what type of drive they were doing or what task they were doing during the drive. There were also no significant differences in standard deviation of driving speed $\left(F(1,37)=2.61, n s, \eta^{2}=\right.$ $.07)$
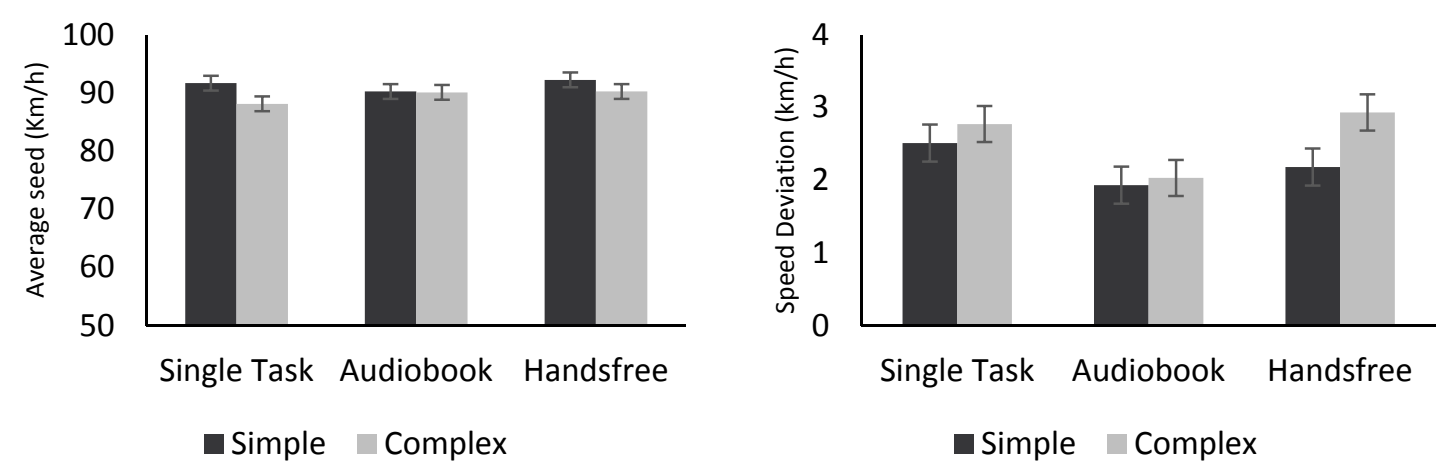

Figure 2. Average speed and standard deviation of speed as a function of task condition and road type. Standard error bars included

Hazard RT were measured as the time between the emergence of the hazard and the participant's first application of the brake. Before analysis, RT were screened for outliers. Specifically, for each driver, RT more than 2.5 SD away from that individual's respective mean in that condition were dropped (1.1\% of the RT). Results are presented in Figure 3. Mixed factorial analyses of variance indicated main effects of both road type $\left(F(1,37)=9.89, p<.01, \eta^{2}=.21\right)$ and task condition $\left(F(2,36)=4.92, p<.01, \eta^{2}=.12\right)$, as well as a Road type $\mathrm{X}$ Task condition interaction $\left(F(2,36)=4.62, p<.01, \eta^{2}=.11\right)$ Post hoc tests revealed that in the complex drive, there were no significant different differences in hazard response times as a function of task condition. However, as predicted, for simple drives, participants braked faster to hazards when they were driving while listening to audiobooks than in the other two conditions $(p<.05)$. 
As for steering performance, the results were unexpected. Although there were curved sections on the complex road, steering performance was always measured in straight sections of the drive. Analyses revealed a Task Load X Drive Type interaction $\left(F(1,37)=6.94, p<.01, \eta^{2}=.16\right)$. Interestingly, in the complex drives, post-hoc analyses revealed was no significant differences in the effects of task condition. However, in the simple drives, steering performance was significantly worse in the audiobook than hands-free condition $(p<.001)$. Interestingly, there was a trend towards higher SDLP on the simple than complex drives in both the single-task driving and audiobook conditions, though the difference was only significant in the audiobook condition. It is possible that on the simple drives, the steering stability deteriorated because there was so little traffic and no turns. The challenge of the drive was too low, and this may have contributed to more erratic steering due to "under-load", though if that was the case, the effect should have been stronger in the single task than audiobook condition.
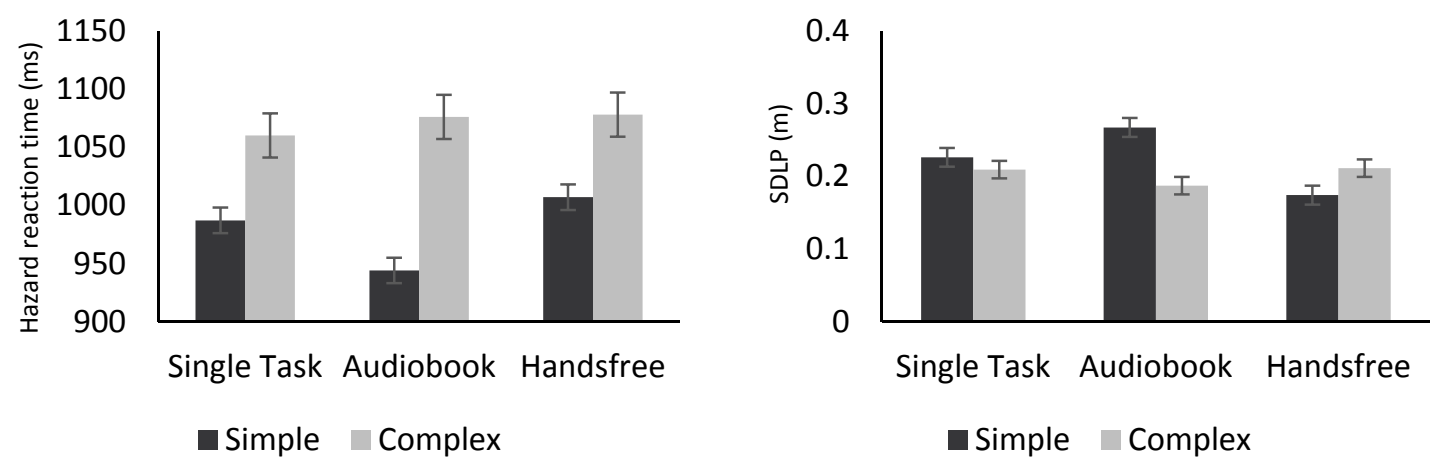

Figure 3. Hazard response times (HRT) and Standard Deviation of Lateral Position (SDLP) as a function of task condition and road type. Standard error bars included

\section{CONCLUSIONS}

Overall, this study has made a contribution in that it showed that there can be beneficial effects of some types of secondary tasks in some types of driving environment, at least as measured by hazard response. When drivers traversed a road of low complexity, with little scenery or oncoming traffic, hazard response times were significantly faster for those listening to audiobooks than it was for those who were just driving, or driving while using a hands-free phone. This result may be interpreted as evidence that in the simple drive, arousal levels were low due to low levels of cognitive load. In such cases, listening to the audiobooks increased the driver's cognitive load to a more optimal level, leading to faster hazard RT. If this occurred, this would suggest that there are occasions where having a little distraction (in the form of an audiobook) may help a driver stay focused. However, it is also important to note that if the secondary task is more demanding (as in a hands-free conversation) or the drive more challenging, secondary tasks are not beneficial. This suggests that a more nuanced approach to the problem of driver distraction is required, one that takes into account both the nature of the distraction and the nature of the driving environment.

This study is just a preliminary investigation though, and as such, it has several limitations. First, it would be better if the drives were longer and it would also be useful if we could truly 
investigate the effects of routine driving, by having drivers repeatedly traverse the same roads. As it is, it is possible that the driving alone condition did not elicit enough boredom/ mindwandering to produce an effect. Second, the audiobook was "Harry Potter", a children's book that is familiar to many. It is possible that a book that was more difficult to understand would interfere more with driving. Furthermore, because the book was familiar, it is possible that the drivers did not pay close attention to the book. A post-drive measure of comprehension would have been a beneficial. It would have also been beneficial to include self-report measures of workload such as the NASA-TLX after each drive as well as post-drive measures of driver boredom and mind-wandering. Finally, because the simple and complex drives differ in terms of traffic, road curvature, and scenery, it is unclear which of these three factors is most important in determining when secondary tasks such as audiobooks begin to interfere.

In addition, it is important to investigate the role that individual differences play. For example, Watson and Strayer (2010) identified individual they called "Super-taskers", individuals with better executive working memory capacity than most as measured by tasks such as the Operation Span. It is possible that the deleterious effects of boredom, which reflects cognitive under-load, may be especially acute for those with more cognitive resources. These individuals may benefit most (or suffer less) as the result of a low-grade secondary task such as listening to an audiobook. It is probable that both individual and situational factors are important in determining if and when secondary tasks can be beneficial in helping drivers stay focused on routine drives. We are currently completing a more comprehensive experiment in which the above limitations are being addressed. Specifically, we have designed new terrains where roadside scenery is manipulated independent of traffic density to better understand the unique contribution of each variable. Furthermore, we ask participants whether they have read the book, and have constructed additional questionnaires to address participant comprehension amongst other factors.

\section{REFERENCES}

Bergmark, R. W., Gliklich, E., Guo, R., Gliklich, R. E., Atchley, P., Atwood, S., ... Christoforou, C. (2016). Texting while driving: the development and validation of the distracted driving survey and risk score among young adults. Injury Epidemiology, 3(1), 7. https://doi.org/10.1186/s40621-016-0073-8

Dahlen, E. R., Martin, R. C., Ragan, K., \& Kuhlman, M. M. (2005). Driving anger, sensation seeking, impulsiveness, and boredom proneness in the prediction of unsafe driving. Accident; Analysis and Prevention, 37(2), 341-8. https://doi.org/10.1016/j.aap.2004.10.006

Horberry, T., Anderson, J., Regan, M. A., Triggs, T. J., \& Brown, J. (2006). Driver distraction: the effects of concurrent in-vehicle tasks, road environment complexity and age on driving performance. Accident; Analysis and Prevention, 38(1), 185-91. https://doi.org/10.1016/j.aap.2005.09.007 
Lee, V. K., Champagne, C. R., \& Francescutti, L. H. (2013). Fatal distraction: Cell phone use while driving. Can Fam Physician, 59(7), 723-725. Retrieved from http://www.cfp.ca/content/59/7/723.full

Lee, Y.C., Lee, J. D., \& Boyle, L. N. (2007). Visual attention in driving: the effects of cognitive load and visual disruption. Human Factors, 49(4), 721-33. Retrieved from http://www.ncbi.nlm.nih.gov/pubmed/17702223

Matthews, G., \& Desmond, P. A. (2002). Task-induced fatigue states and simulated driving performance. The Quarterly Journal of Experimental Psychology. A, Human Experimental Psychology, 55(2), 659-86. https://doi.org/10.1080/02724980143000505

Palinko, O., \& Kun, A. L. (2012). Exploring the effects of visual cognitive load and illumination on pupil diameter in driving simulators. In Proceedings of the Symposium on Eye Tracking Research and Applications - ETRA'12 (p. 413). New York, New York, USA: ACM Press. https://doi.org/10.1145/2168556.2168650

Paxion, J., Galy, E., \& Berthelon, C. (2015). Overload depending on driving experience and situation complexity: Which strategies faced with a pedestrian crossing? Applied Ergonomics, 51, 343-9. https://doi.org/10.1016/j.apergo.2015.06.014

Schroeter, R., Oxtoby, J., \& Johnson, D. (2014). AR and Gamification Concepts to Reduce Driver Boredom and Risk Taking Behaviours. In Proceedings of the 6th International Conference on Automotive User Interfaces and Interactive Vehicular Applications AutomotiveUI'14 (pp. 1-8). New York, New York, USA: ACM Press. https://doi.org/10.1145/2667317.2667415

Schwarz, J. F. A., Ingre, M., Fors, C., Anund, A., Kecklund, G., Taillard, J., ... Åkerstedt, T. (2012). In-car countermeasures open window and music revisited on the real road: popular but hardly effective against driver sleepiness. Journal of Sleep Research, 21(5), 595-9. https://doi.org/10.1111/j.1365-2869.2012.01009.x

Strayer, D. L., Cooper, J. M., Turrill, J., Coleman, J. R., Medeiros-Ward, N. N., \& Biondi, F. (2015). Measuring Cognitive Distraction in the Automobile. Human Factors, 57(8), 1300 24. https://doi.org/10.1177/0018720815575149 